\title{
Gray Matter Volume Reductions Were Associated with TPH1 Polymorphisms in Depressive Disorder Patients with Suicidal Attempts
}

\author{
Sang Min Lee ${ }^{1}$, Soyoen Lee ${ }^{2}$, Won Sub Kang ${ }^{1}$, Geon-Ho Jahng ${ }^{3}$, \\ Hae Jeong Park ${ }^{4}$, Su Kang Kim ${ }^{5}$ and Jin Kyung Park ${ }^{1 凶}$ \\ 'Department of Psychiatry, School of Medicine, Kyung Hee University, Seoul, Republic of Korea \\ 2Department of Medicine, Graduate School, Kyung Hee University, Seoul, Republic of Korea \\ ${ }^{3}$ Department of Radiology, Kyung Hee University Hospital at Gangdong, College of Medicine, Kyung Hee University, Seoul, Republic of Korea \\ ${ }^{4}$ Department of Pharmacology, School of Medicine, Kyung Hee University, Seoul, Republic of Korea \\ ${ }^{5}$ Department of Biomedical Laboratory Science, Catholic Kwandong University, Gangneung, Republic of Korea
}

Objective Structural changes of brain areas have been reported in depressive disorder and suicidal behavior (SB), in which TPH1 also has been known as a promising candidate gene. We investigated gray matter volume (GMV) differences, TPH1 rs1800532 and rs1799913 polymorphisms previously found to be associated with depressive disorder and SB, and the relationship between the two markers.

Methods Thirteen depressive disorder patients with suicidal attempts (SA) and twenty healthy controls were included. We examined GMV differences using a voxel-based morphometry and regions of interest analysis. Direct sequencing was used for genotyping.

Results The patients showed significant GMV reduction in left cerebral region including middle frontal gyrus, inferior frontal gyrus, and anterior cingulate cortex; in right middle temporal gyrus; in left cerebellar tonsil; and in right cerebral region including precentral gyrus and postcentral gyrus (corrected $\mathrm{p}<0.005$ ). The right precentral and postcentral gyri GMV values of AA and CA genotypes patients were significantly decreased compared to those of CC genotype subjects (corrected $\mathrm{p}=0.040$ ).

Conclusion These findings show the possibility that both GMV reductions and TPH1 rs1800532/rs1799913 A allele may be involved in the pathogenesis of depressive disorder patients with SA.

Psychiatry Investig 2018;15(12):1174-1180

Key Words Depressive disorder, Suicidal attempt, Gray matter volume, TPH1 gene.

\section{INTRODUCTION}

As one of the most common psychiatric disorders, depression is a major cause of disability worldwide. According to the World Health Organization's (WHO) statistics, more than 300 million people worldwide suffer from depression. ${ }^{1}$ However, its precise neurobiological mechanisms are not known yet. ${ }^{2}$ Recently, many neuroimaging studies using magnetic resonance imaging (MRI) have elucidated disorder-related patterns of structural changes in brain associated with depressive disorder. Particularly several regional gray matter changes in

Received: June 9, 2018 Revised: October 27, 2018

Accepted: November 1, 2018

$\triangle$ Correspondence: Jin Kyung Park, MD, PhD

Department of Psychiatry, Kyung Hee University Hospital at Gangdong, 892 Dongnam-ro, Gangdong-gu, Seoul 05278, Republic of Korea Tel: +82-2-440-6175, Fax: +82-2-440-6073, E-mail: parkdawit@naver.com

(a) This is an Open Access article distributed under the terms of the Creative Commons Attribution Non-Commercial License (https://creativecommons.org/licenses/bync/4.0) which permits unrestricted non-commercial use, distribution, and reproduction in any medium, provided the original work is properly cited. frontal lobe, temporal lobe, hippocampus, amygdala, thalamus, and striatum related to mood regulation and cognitive function have supported the neurobiological basis on many psychological and behavioral symptoms of depressive disorder. ${ }^{3-5}$

Suicide is a leading cause of death worldwide, and becomes a significant public health problem. ${ }^{1}$ Sixty percent of people who committed suicide had a history of depressive disorder. ${ }^{6}$ Although the close relationship between suicidal behaviors (SB) and depressive disorder has been well known, the underlying mechanisms between them have been suggested inconsistently. Previous studies investigating SB in depressive disorder patients reported local gray matter changes of several cortical and subcortical structures. ${ }^{7-10}$ These findings suggest the possibility that the development of SB in depressive disorder may be associated with structural abnormalities of the brain.

There is growing evidence that genetic factors are involved in the pathophysiology both of depressive disorder and SB. Genetic variants of TPH1, a gene encoding the key enzyme 
tryptophan hydroxylase 1 (TPH1) in serotonin synthesis are ones of the most studied in genetic researches concerning depression and suicide. ${ }^{11}$ The most investigated single nucleotide polymorphism (SNP) rs1800532 of TPH1 was reported to be associated with the occurrence of depression. ${ }^{12-14}$ Bellivier et al. ${ }^{12}$ reported a significant contribution of A allele in rs 1800532 to SB from a meta-analysis of 9 studies. TPH1 SNP rs1799913 also has been studied along with rs1800532 to be known as a risk variant of depression and SB. The rs1800532/ rs1799913 polymorphisms were shown as strongly associated with SB in both European and Asian populations. ${ }^{15}$

Given the assumption that both structural changes of brain and genetic polymorphisms are involved in the pathogenesis of depression and SB simultaneously, we sought to investigate gray matter volume (GMV) differences, TPH1 polymorphisms, and the relationship between the two potential biomarkers in depressive disorder patients with suicidal attempts (SA). The authors performed voxel-based morphometry to detect regions of differential GMV. Based on these results, the regions of interest (ROIs) were selected, and the GMV differences of these ROIs were analyzed to investigate correlations with behavioral data related to suicide. Then the association between GMV differences and genotype data of rs1800532/rs1799913 was tested.

\section{METHODS}

\section{Subjects}

Subjects were recruited from the department of psychiatry of Kyung Hee University Hospital at Gangdong, Seoul, Republic of Korea. Thirteen individuals with the diagnosis of depressive disorder were included. The diagnosis was determined according to ICD-10 criteria based on the structured interview by board-certified psychiatrists. All of them had history of $\mathrm{SA}$, which were defined as any act carried out with a certain intent to die, and confirmed by others or psychiatric specialists in clinical settings. The patients group consisted of $12 \mathrm{fe}-$ males and 1 male, and was aged $20-47$ years ( $31.9 \pm 10.0$ years, mean age \pm standard deviation). Twenty healthy controls were without current or past diagnosis of psychiatric disorder, and had no history of SA. The control group consisted of 13 females and 7 males, and was aged $22-48$ years ( $33.6 \pm 6.2$ years). Exclusion criteria were neurological disorders, serious head injury, mental retardation, severe somatic diseases, or contraindication to MRI scanning.

All subjects were assessed with the Scale for Suicide Ideation (SSI) ${ }^{16}$ the Early Trauma Inventory Self Report-Short Form (ETISR-SF), ${ }^{17}$ the Barratt Impulsiveness Scale (BIS-11), ${ }^{18}$ and the Beck Hopelessness Scale (BHOP) ${ }^{19}$ For continuous variables, independent samples t-test was used, and for categorical variables, Pearson's chi-squared test was used to test the differences between the two groups. Written informed consent was obtained from each subject. This study was conducted according to the guidelines of the Helsinki Declaration, and was approved by the Institutional Review Board of Kyung Hee University Hospital at Gangdong (KHNMC IRB 2012-028).

\section{MRI data acquisition}

Images were acquired with a 3.0 Tesla MRI scanner (Achieva, Philips Medical Systems, Best, The Netherlands) using a dedicated 8-element phased array sensitivity-encoding head coil. A three-dimensional T1-weighted sagittal images (3DT1WI) were acquired with the magnetization-prepared rapid acquisition of gradient echo (MPRAGE) sequence with the following imaging parameters: $\mathrm{TR}=9.9 \mathrm{~mm}, \mathrm{TE}=4.6 \mathrm{~ms}$, flip angle $=8^{\circ}$, FOV $=240 \times 240 \mathrm{~mm}$, slice thickness $=1 \mathrm{~mm}$, matrix size $=240 \times$ 240 , and resolution $=1.00 \times 1.00 \times 1.00 \mathrm{~mm}$. In addition, anatomical images were acquired with a fluid-attenuated inversion recovery (FLAIR) images (TR=11000 ms, TE=125 ms, $\mathrm{TI}=2800 \mathrm{~ms}$ ) for finding structural abnormalities.

\section{MRI data analysis}

Statistical Parametric Mapping 12 (SPM12, Wellcome Department of Cognitive Neurology, London, UK) (http://www. fil.ion.ucl.ac.uk/spm/) was used for pre-processing of MRI data with the following steps. First, MRI images were realigned to minimize the subject motion during the scan. Second, the realigned MRI images and the 3D T1WI for each subject were co-registered. Third, the images were segmented into gray matter, white matter, and others including CSF. Then the 3D T1WI was spatially normalized into the Montreal Neurological Institute (MNI) standard anatomical space, ${ }^{20}$ and MRI images were also standardized with information of the normalization factor obtained from the 3D T1WI. Finally, the spatially normalized fMRI data were smoothed by $8 \times 8 \times 8$ $\mathrm{mm}$ of the full within half maximum (FWHM) of the Gaussian filter to obtain 3D T1WI tissue volume maps.

To compare GMVs between the two groups, voxel-wise, one-way analysis of variance (ANOVA) tests with subject's total intra-cranial volume and age as covariates were used. The threshold of $p$ value was set at 0.001 without performing the correction of the multiple comparison with an extent threshold of 20 voxels. This extent threshold was chosen to be small enough to capture a small structure but not so small as to capture false positives. All local maxima were represented as Talairach coordinates. In addition, the cluster size and the peak $\mathrm{T}$ value of areas of significant increase were given. Relevant anatomical landmarks and Brodmann's areas (BA) were identified using GingerALE (http://www.brainmap.org), and analyzed step by step using Talairach Client (http://www.talairach. org). Then ROIs were chosen based on the significant results 
of voxel-based comparison between the two groups. The ROIs were fixed using Automated Anatomical Labeling and wfu_ pickatlas toolbox for SPM12. The GMV values of each ROI were measured using MRIcroN. Differences of GMVs from each ROI between the two groups were assessed using t-test. To avoid chance findings due to multiple testing, Bonferroni correction was applied.

\section{SNP genotyping}

Genomic DNA was isolated from the whole blood of all subjects using the High Pure PCR Template Preparation kit (Roche, Mannheim, Germany). SNP genotyping was conducted using direct sequencing using the following primers for both rs1800532 and rs1799913 (sense,5'-GCCAGGAATTCATCAAATGG-3'; antisense,5'-CAAGCCAATTTCTTGGGAGA-3'). All PCR products were sequenced using an ABI PRISM 3730XL analyzer (PE Applied Biosystems, Foster City, CA, USA). Sequence data were analyzed using SeqManII software (DNASTAR Inc., Madison, WI, USA). Hardy-Weinberg equilibrium (HWE) was performed using SNPStats (http://bioinfo.iconcologia.net/snpstats/start.htm). Haploview 4.2 (Broad Institute, Cambridge, MA, USA) was used to calculate the pairwise linkage disequilibrium (LD) between the two SNPs. Pearson's chi-squared test was used to test for group differences of genotype and allele frequencies.

\section{Relationship among GMV, behavioral, and genotype data}

Each patient's GMV values were extracted for each ROI obtained by the procedures described above. Correlation matrices were used to evaluate the relationship between the GMV and the clinical variables (SSI, ETISR-SF, BIS-11, and BHOP) in the patients. The normality of the data was assessed using Shapiro-Wilk test, and Spearman's rank correlation tests were applied on the parameters. In order to investigate the relationship between GMV differences and alleles, differences of GMV values according to genotypes (minor allele homozygotes and heterozygotes vs. major allele homozygotes; minor allele homozygotes vs. heterozygotes and major allele homozygotes) were compared using an independent samples t-test. All statistical analysis was performed using R version 3.4.3 (https://cran. r-project.org/), and Bonferroni correction was applied.

\section{RESULTS}

\section{Behavioral data}

Table 1 summarizes the characteristics and behavioral data of the subjects. Age $(p=0.556)$ and gender $(p=0.169)$ were not significantly different between the two groups. The subjective ratings for SSI, ETISR-SF, BIS-11, and BHOP were obtained from 30 subjects, but not from 3 subjects of the controls denied to report those. The scores of SSI $(\mathrm{p}<0.001)$, ETISR-SF ( $\mathrm{p}=$ $0.005)$, and BHOP $(\mathrm{p}<0.001)$ were significantly much higher in the patients than those in the controls analyzed by using $\mathrm{t}$ test. There was no significant difference in the scores of BIS11 between the two groups ( $\mathrm{p}=0.58)$. A lowered significance level of $\mathrm{p}$ value to 0.006 ( $=0.05 / 8$ for the four behavioral variables and the two groups) by Bonferroni correction was applied to this comparison.

\section{MRI data}

Compared to the controls, the patients showed reduced GMV in the left cerebral region including middle frontal gyrus (BA 10), inferior frontal gyrus, and anterior cingulate cortex (BA 32); in the right middle temporal gyrus (BA 21); in the left cerebellar tonsil; and in the right cerebral region including precentral gyrus (BA 4) and postcentral gyrus [BA 3, ANOVA, $\mathrm{df}=(1.0,30.0), \mathrm{p}<0.001]$. These regions were selected for ROIs (Figure 1). ROI 1 consisted of left middle frontal gyrus, inferior frontal gyrus, and anterior cingulate cortex; ROI 2, right middle temporal gyrus; ROI 3, left cerebellar tonsil; and ROI 4, right precentral gyrus and postcentral gyrus. All the GMV values extracted from each ROI were significantly decreased in the patients than those in the controls $(\mathrm{p}<0.001)$ (Table 1). The p value of 0.006 (=0.05/8 for the four ROIs and the two groups) was applied to a significant threshold.

\section{Genotype data}

Genotype distributions of both rs180052 and rs1799913 in the controls and the patients groups were in HWE. The individuals included in the counts of genotypes and alleles for the two SNPs were same subjects. With the finding that the two SNPs were in LD ( $\left.\left|\mathrm{D}^{\prime}\right|=1.0, \mathrm{r}^{2}=0.941\right)$ indicating strong association between the alleles, we considered the two SNPs as one for the correction of multiple comparisons. For both SNPs, the genotype and allele frequencies were not significantly different between the two groups (rs1800532: genotype, $\chi^{2}=3.540, \mathrm{df}=2, \mathrm{p}=0.170$, allele, $\chi^{2}=2.128, \mathrm{df}=1, \mathrm{p}=0.145$; rs1799913, genotype, $\chi^{2}=4.357, \mathrm{df}=2, \mathrm{p}=0.113$, allele, $\chi^{2}=2.754$, $\mathrm{df}=1, \mathrm{p}=0.097)$.

\section{Relationship between GMV and behavioral data}

A correlation was found between BIS-11 scores and the ROI 3 GMV values $(r=0.647, p=0.017)$ in the patients. However, it did not remain significant after Bonferroni correction. No significant correlation was found between other behavioral data and other ROIs GMV values.

\section{Relationship between GMV and genotype data}

For investigating the GMV differences according to allele, 
Table 1. Characteristics, behavioral data, gray matter volume values from four regions of interest, and genotype data of participants

\begin{tabular}{|c|c|c|c|}
\hline & Patients $(\mathrm{N}=13)$ & Controls $(\mathrm{N}=20)$ & $\mathrm{p}$ value \\
\hline Gender (female/male) & $12 / 1$ & $13 / 7$ & 0.169 \\
\hline Age & $31.9(10.0)$ & $33.6(6.2)$ & 0.556 \\
\hline SSI & $20.7(9.3)$ & $1.7(1.6)$ & $<0.001$ \\
\hline ETISR-SF & $8.4(5.3)$ & $3.5(3.4)$ & 0.005 \\
\hline BIS-11 & $29.7(8.1)$ & $28.3(4.5)$ & 0.581 \\
\hline $\mathrm{BHOP}$ & $12.7(5.8)$ & $3.1(2.4)$ & $<0.001$ \\
\hline \multicolumn{4}{|l|}{ GMV } \\
\hline ROI 1 & $0.410(0.028)$ & $0.482(0.035)$ & $<0.001$ \\
\hline ROI 2 & $0.395(0.034)$ & $0.454(0.037)$ & $<0.001$ \\
\hline ROI 3 & $0.537(0.060)$ & $0.643(0.069)$ & $<0.001$ \\
\hline ROI 4 & $0.269(0.041)$ & $0.325(0.039)$ & $<0.001$ \\
\hline \multicolumn{4}{|l|}{ rs1800532 } \\
\hline Genotype & & & 0.170 \\
\hline $\mathrm{CC}$ & 4 & 3 & \\
\hline CA & 8 & 10 & \\
\hline $\mathrm{AA}$ & 1 & 7 & \\
\hline Allele & & & 0.145 \\
\hline $\mathrm{C}$ & 0.615 & 0.4 & \\
\hline $\mathrm{A}$ & 0.385 & 0.6 & \\
\hline \multicolumn{4}{|l|}{ rs1799913 } \\
\hline Genotype & & & 0.113 \\
\hline CC & 4 & 3 & \\
\hline CA & 8 & 9 & \\
\hline AA & 1 & 8 & \\
\hline Allele & & & 0.097 \\
\hline $\mathrm{C}$ & 0.615 & 0.375 & \\
\hline A & 0.385 & 0.625 & \\
\hline
\end{tabular}

Frequencies are presented for gender, genotype, and allele. Difference in these variables between two groups were analyzed with chi-square test. Mean values and standard deviations (in brackets) are presented for age. SSI: Scale for Suicide Ideation, ETISR-SF: Early Trauma Inventory Self Report-Short Form, BIS-11: Barratt Impulsiveness Scale, the Beck Hopelessness Scale (BHOP), and gray matter volume values (GMV) from region of interest (ROI 1; left middle frontal gyrus, inferior frontal gyrus, and anterior cingulate cortex); ROI 2 (right middle temporal gyrus); ROI 3 (left cerebellar tonsil); and ROI 4 (right precentral and postcentral gyri). Differences in these variables between two groups were analyzed with t-test

we compared the GMV values from 4 ROIs between A-carriers (AA and CA genotypes) and CC genotype subjects in the patients (Table 2). For both rs180052 and rs1799913, the ROI 4 GMV values of A carriers were significantly decreased compared to those of CC genotype subjects in the patients ( $\mathrm{p}=$ 0.010 ) (Figure 2). The significance level of $\mathrm{p}$ value was 0.013 ( $=0.05 / 4$ for the four ROIs). No difference was found for other ROIs GMV values between the two groups. Then we tried to analyze the GMV differences from 4 ROIs between C-carriers (CC and CA genotypes) and AA genotype subjects. However, we were not able to perform statistical analyses in the patients because the number of subject with AA genotype was one for both SNPs. In the controls, we found no difference of
4 ROIs GMV values between C-carriers and AA genotype subjects for both SNPs (data not shown).

\section{DISCUSSION}

This is a study attempting to elucidate neurobiological mechanisms underlying depressive disorder with SB. A link between GMV differences and genetic polymorphisms was investigated. The patients showed significant GMV reductions in the left cerebral region including middle frontal gyrus (BA $10)$, inferior frontal gyrus, and anterior cingulate cortex (BA 32). GMV reductions in the middle frontal gyrus are consistent with previous studies with depressive disorder patients. ${ }^{5,21,22}$ 

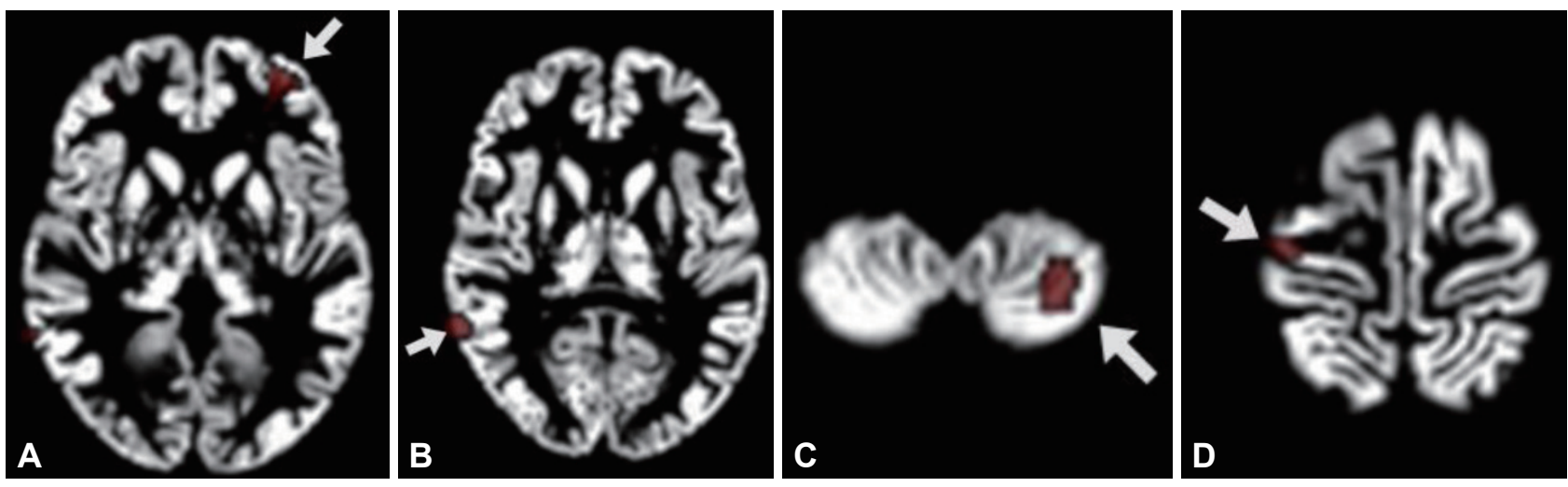

Figure 1. Regions of interest (ROIs) to be shown as significant gray matter volume reduction in the patients compared to the controls. (A) is ROI 1 including left middle frontal gyrus $(x=-28, y=48, z=-1)$, inferior frontal gyrus $(x=-38, y=44, z=-7)$, and anterior cingulate cortex $(x=-26$, $y=44, z=4)$; $(B)$ is ROI 2, right middle temporal gyrus $(x=63, y=-47, z=8)$; (C) is ROI 3, left cerebellar tonsil $(x=-28, y=-57, z=-49)$; and (D) is ROI 4 including right precentral gyrus $(x=37, y=-17, z=66)$ and postcentral gyrus $(x=33, y=-23, z=70)$.

Table 2. Gray matter volume values from four regions of interest between A-carriers and CC

\begin{tabular}{cccc}
\hline Subjects & AA, CA $(\mathrm{N}=9)$ & $\mathrm{CC}(\mathrm{N}=4)$ & $\mathrm{p}$ value \\
\hline rs1800532 & & & \\
ROI 1 & $0.403(0.028)$ & $0.425(0.025)$ & 0.212 \\
ROI 2 & $0.388(0.039)$ & $0.409(0.017)$ & 0.326 \\
ROI 3 & $0.554(0.062)$ & $0.498(0.031)$ & 0.124 \\
ROI 4 & $0.251(0.028)$ & $0.310(0.039)$ & 0.010 \\
rs1799913 & & & \\
ROI 1 & $0.403(0.028)$ & $0.425(0.025)$ & 0.212 \\
ROI 2 & $0.388(0.039)$ & $0.409(0.017)$ & 0.326 \\
ROI 3 & $0.554(0.062)$ & $0.498(0.031)$ & 0.124 \\
ROI 4 & $0.251(0.028)$ & $0.310(0.039)$ & 0.010 \\
\hline
\end{tabular}

Mean values and standard deviations (in brackets) are presented. Differences in these variables between AA, CA and CC groups were analyzed using t-test with a significant threshold of $\mathrm{p}<0.013$ after Bonferroni correction. Region of interest (ROI) 1 is left middle frontal gyrus, inferior frontal gyrus, and anterior cingulate cortex; ROI 2, right middle temporal gyrus; ROI 3, left cerebellar tonsil; and ROI 4, right precentral and postcentral gyri

Abnormalities in activity of the middle frontal gyrus were reported in functional studies of depression. ${ }^{23,24}$ Altered functional connectivity between the middle frontal gyrus and other regions such as insula and anterior cingulate cortex was also found in depression. ${ }^{25,26}$ Taken together, changes in the middle frontal gyrus GMV could be essential to the pathophysiology of depression, particularly to emotional processing.

In the analysis among ROIs GMV and behavioral data, we found a trend of correlation between BIS-11 scores and the left cerebellar tonsil GMV values in the patients. Impulsivity is one of the most studied endophenotypes in suicide researches. ${ }^{27,28}$ It was identified as a risk factor associated with SA in depression..$^{29,30}$ The cerebellum has been known as related traditionally to motor functions. However, recent findings showed that it was also related to the higher cortical functions
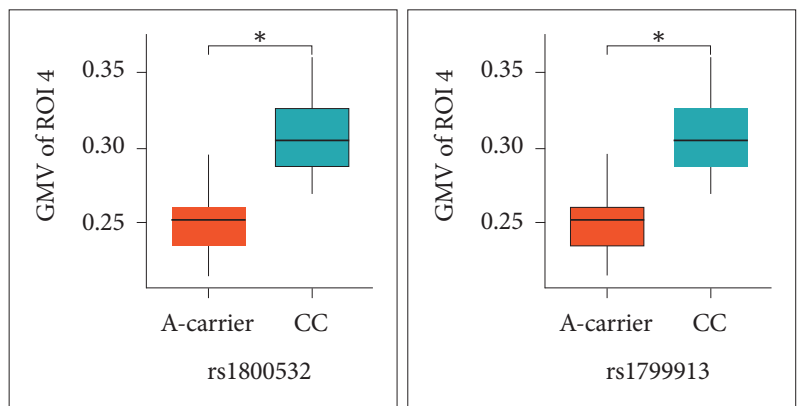

Figure 2. Comparison of gray matter volume (GMV) values in the right precentral and postcentral gyri between A-carriers and CC genotype subjects in the patients. Differences were analyzed with t-test. *corrected $p$ value by Bonferroni method is 0.040 . GMV of region of interest $4(\mathrm{ROI} 4)$ is gray matter volume values of the right precentral and postcentral gyri; A-carrier, the subjects with $\mathrm{AA}$ and $\mathrm{CA}$ genotypes; $\mathrm{CC}$, the subjects with $\mathrm{CC}$ genotype.

such as cognition, learning, memory, and prediction. ${ }^{31}$ While the neurobiological mechanisms of impulsivity related to the cerebellum have not been known, our results may suggest an evidence of cerebellar function involvement in the neurobiology of impulsivity.

Recently, several neuroimaging findings have supported the potential role of the precentral gyrus in neurobiological understandings of depression and suicide. Decreased cortical thickness in the precentral gyrus was noted in major depressive disorder patients. ${ }^{32} \mathrm{~A}$ strong association between accelerated cortical thinning of precentral regions and depression symptomatology was observed in adolescents. ${ }^{33}$ In a functional neuroimaging study, suicidal attempters with depressive disorder exhibited a reduced precentral regional response than those without SA. ${ }^{34}$ Minzenberg et al. ${ }^{35}$ reported that past $\mathrm{SB}$ was associated with higher control related activation in several brain regions including precentral gyrus in mood disorder patients. The precentral gyrus is a part of the frontal lobe that consists primarily of the primary motor cortex, and it is involved in the task of controlling the execution of the re- 
sponse inhibition. ${ }^{36,37}$ Disabilities in inhibition control are associated with a more propensity to act on suicidal feelings. ${ }^{38}$ These findings suggest that the precentral gyrus may be an important role in impulsivity regulation related to SB.

Consistent with the previous reports, we observed that the GMV values in the ROI 4 consisted of right precentral and postcentral gyri were significantly decreased in the depressed patients with SA than in the controls. Moreover, for both rs1800532 and rs1799913, the ROI 4 GMV values of A carriers were significantly reduced compared to those of CC genotype subjects in the patients. Mirkovic et al. ${ }^{39}$ reported a strong overall association of the two SNPs with SB, and suggested that the A allele increased a risk of SB in dose-dependent manner. Taken together, our results suggest that A allele may modulate the structural changes in the precentral gyrus, which affect suicidal acts in the depressive disorder patients.

The limitation of this study is relatively small sample size to be verified in the further study with larger samples. Other limitations are as follows: diagnoses were not assessed with Structured Clinical Interview for DSM Disorders, the depressed patients who did not attempt suicide were not included in the control group, and there are needs to investigate other variants in TPH1 and to perform further molecular biologic studies for elucidating their functions. With an attempt to identify a link between cerebral structural changes and genetic polymorphisms observed in the depressive disorder patients with SA, our findings suggest an evidence of neurobiological mechanisms underlying these patients.

In conclusion, we found significant GMV reductions in several regions of frontal lobe, temporal lobe, paracentral gyrus, and cerebellum in depressive disorder patients with SA. The paracentral gyrus GMV reduction was associated with A carriers of rs1800532/rs1799913 in the patients. These results suggest that GMV differences and TPH1 polymorphisms may be simultaneously implicated in the pathogenesis of depression with SB.

\section{Acknowledgments}

This study was supported by a grant from National Research Foundation of Korea (NRF-2012R1A1A1012760).

\section{REFERENCES}

1. World Health Organization. Depression and Other Common Mental Disorders: Global Health Estimates. Geneva: World Health Organization; 2017.

2. Zhang FF, Peng W, Sweeney JA, Jia ZY, Gong QY. Brain structure alterations in depression: psychoradiological evidence. CNS Neurosci Ther 2018;24:994-1003.

3. van Eijndhoven P, van Wingen G, Katzenbauer M, Groen W, Tepest R, Fernandez G, et al. Paralimbic cortical thickness in first-episode depression: evidence for trait-related differences in mood regulation. Am J Psychiatry 2013;170:1477-1486.

4. Jia Z, Wang Y, Huang X, Kuang W, Wu Q, Lui S, et al. Impaired fronto- thalamic circuitry in suicidal patients with depression revealed by diffusion tensor imaging at 3.0 T. J Psychiatry Neurosci 2014;39:170-177.

5. Peng W, Chen Z, Yin L, Jia Z, Gong Q. Essential brain structural alterations in major depressive disorder: a voxel-wise meta-analysis on first episode, medication-naive patients. J Affect Disord 2016;199:114-123.

6. Lee BH, Kim YK. Potential peripheral biological predictors of suicidal behavior in major depressive disorder. Prog Neuropsychopharmacol Biol Psychiatry 2011;35:842-847.

7. Ding Y, Lawrence N, Olie E, Cyprien F, le Bars E, Bonafe A, et al. Prefrontal cortex markers of suicidal vulnerability in mood disorders: a model-based structural neuroimaging study with a translational perspective. Transl Psychiatry 2015;5:e516.

8. Hwang JP, Lee TW, Tsai SJ, Chen TJ, Yang CH, Lirng JF, et al. Cortical and subcortical abnormalities in late-onset depression with history of suicide attempts investigated with MRI and voxel-based morphometry. J Geriatr Psychiatry Neurol 2010;23:171-184.

9. Monkul ES, Hatch JP, Nicoletti MA, Spence S, Brambilla P, Lacerda $\mathrm{AL}$, et al. Fronto-limbic brain structures in suicidal and non-suicidal female patients with major depressive disorder. Mol Psychiatry 2007; 12:360-366.

10. Peng H, Wu K, Li J, Qi H, Guo S, Chi M, et al. Increased suicide attempts in young depressed patients with abnormal temporal-parietal-limbic gray matter volume. J Affect Disord 2014;165:69-73.

11. Mann JJ, Arango V, Underwood MD. Serotonin and suicidal behavior. Ann N Y Acad Sci 1990;600:476-484; discussion 484-485.

12. Bellivier F, Chaste P, Malafosse A. Association between the TPH gene A218C polymorphism and suicidal behavior: a meta-analysis. Am J Med Genet B Neuropsychiatr Genet 2004;124B:87-91.

13. Jokela M, Raikkonen K, Lehtimaki T, Rontu R, Keltikangas-Jarvinen L. Tryptophan hydroxylase 1 gene (TPH1) moderates the influence of social support on depressive symptoms in adults. J Affect Disord 2007; 100:191-197.

14. Wigner P, Czarny P, Synowiec E, Bijak M, Bialek K, Talarowska M, et al. Association between single nucleotide polymorphisms of TPH1 and TPH2 genes, and depressive disorders. J Cell Mol Med 2018;22:17781791.

15. Li D, He L. Further clarification of the contribution of the tryptophan hydroxylase (TPH) gene to suicidal behavior using systematic allelic and genotypic meta-analyses. Hum Genet 2006;119:233-240.

16. Beck AT, Kovacs M, Weissman A. Assessment of suicidal intention: the Scale for Suicide Ideation. J Consult Clin Psychol 1979;47:343-352.

17. Bremner JD, Bolus R, Mayer EA. Psychometric properties of the Early Trauma Inventory-Self Report. J Nerv Ment Dis 2007;195:211-218.

18. Patton JH, Stanford MS, Barratt ES. Factor structure of the Barratt impulsiveness scale. J Clin Psychol 1995;51:768-774.

19. Beck AT, Weissman A, Lester D, Trexler L. The measurement of pessimism: the hopelessness scale. J Consult Clin Psychol 1974;42:861-865.

20. Talairach J, Tournoux P. Co-planar Stereotaxic Atlas of the Human Brain: 3-dimensional Proportional System: an Approach to Cerebral Imaging. G. Thieme. New York: Thieme Medical Publisher; 1988.

21. Abe O, Yamasue H, Kasai K, Yamada H, Aoki S, Inoue H, et al. Voxelbased analyses of gray/white matter volume and diffusion tensor data in major depression. Psychiatry Res 2010;181:64-70.

22. Bell-McGinty S, Butters MA, Meltzer CC, Greer PJ, Reynolds CF 3rd, Becker JT. Brain morphometric abnormalities in geriatric depression: long-term neurobiological effects of illness duration. Am J Psychiatry 2002;159:1424-1427.

23. Feeser M, Schlagenhauf F, Sterzer P, Park S, Stoy M, Gutwinski S, et al. Context insensitivity during positive and negative emotional expectancy in depression assessed with functional magnetic resonance imaging. Psychiatry Res 2013;212:28-35.

24. Liu CH, Ma X, Wu X, Fan TT, Zhang Y, Zhou FC, et al. Resting-state brain activity in major depressive disorder patients and their siblings. J Affect Disord 2013;149:299-306.

25. Connolly CG, Wu J, Ho TC, Hoeft F, Wolkowitz O, Eisendrath S, et al. 
Resting-state functional connectivity of subgenual anterior cingulate cortex in depressed adolescents. Biol Psychiatry 2013;74:898-907.

26. Guo W, Liu F, Xiao C, Zhang Z, Liu J, Yu M, et al. Decreased insular connectivity in drug-naive major depressive disorder at rest. J Affect Disord 2015;179:31-37.

27. Courtet P, Gottesman, II, Jollant F, Gould TD. The neuroscience of suicidal behaviors: what can we expect from endophenotype strategies? Transl Psychiatry 2011;1. pii:e7.

28. Mann JJ, Arango VA, Avenevoli S, Brent DA, Champagne FA, Clayton $\mathrm{P}$, et al. Candidate endophenotypes for genetic studies of suicidal behavior. Biol Psychiatry 2009;65:556-563.

29. Caceda R, Durand D, Cortes E, Prendes-Alvarez S, Moskovciak T, Harvey PD, et al. Impulsive choice and psychological pain in acutely suicidal depressed patients. Psychosom Med 2014;76:445-451.

30. Corruble E, Damy C, Guelfi JD. Impulsivity: a relevant dimension in depression regarding suicide attempts? J Affect Disord 1999;53:211215.

31. Moreno-Rius J, Miquel M. The cerebellum in drug craving. Drug Alcohol Depend 2017;173:151-158.

32. Peng D, Shi F, Li G, Fralick D, Shen T, Qiu M, et al. Surface vulnerability of cerebral cortex to major depressive disorder. PLoS One 2015;10: e0120704.

33. Bos MGN, Peters S, van de Kamp FC, Crone EA, Tamnes CK. Emerg- ing depression in adolescence coincides with accelerated frontal cortical thinning. J Child Psychol Psychiatry 2018;59:994-1002.

34. Tsujii N, Mikawa W, Tsujimoto E, Adachi T, Niwa A, Ono H, et al. Reduced left precentral regional responses in patients with major depressive disorder and history of suicide attempts. PLoS One 2017;12:e0175249.

35. Minzenberg MJ, Lesh TA, Niendam TA, Yoon JH, Cheng Y, Rhoades $\mathrm{RN}$, et al. Control-related frontal-striatal function is associated with past suicidal ideation and behavior in patients with recent-onset psychotic major mood disorders. J Affect Disord 2015;188:202-209.

36. Bush G, Whalen PJ, Rosen BR, Jenike MA, McInerney SC, Rauch SL. The counting Stroop: an interference task specialized for functional neuroimaging--validation study with functional MRI. Hum Brain Mapp 1998;6:270-282.

37. Carter CS, Mintun M, Nichols T, Cohen JD. Anterior cingulate gyrus dysfunction and selective attention deficits in schizophrenia: [15O] H2O PET study during single-trial Stroop task performance. Am J Psychiatry 1997;154:1670-1675

38. Mann JJ. Neurobiology of suicidal behaviour. Nat Rev Neurosci 2003; 4:819-828.

39. Mirkovic B, Laurent C, Podlipski MA, Frebourg T, Cohen D, Gerardin P. Genetic association studies of suicidal behavior: a review of the past 10 years, progress, limitations, and future directions. Front Psychiatry 2016;7:158. 